\title{
Participatory Air Quality and Urban Heat Islands Monitoring System
}

\author{
Mohamed Anis Fekih*, Walid Bechkit*, Hervé Rivano*, Manoël Dahan*, Florent Renard ${ }^{\dagger}$, Lucille Alonso ${ }^{\dagger}$, \\ Florent Pineau ${ }^{\ddagger}$ \\ *Univ Lyon, INSA Lyon, CITI, F-69621 Villeurbanne, France \\ ${ }^{\dagger}$ University Jean Moulin Lyon 3, UMR CNRS Environment City Society, 69007 Lyon, France \\ ¥TOTAL Raffinage-Chimie, Laboratoire de la Qualité de l’Air, 69360 SOLAIZE
}

\begin{abstract}
The widespread use of low-cost environmental monitoring systems, together with recent developments in the design of Internet of Things architectures and protocols, has given new impetus to smart city applications. Such progress should, in particular, considerably improve the fine characterization of a wide range of physical quantities within our cities. Indeed, the cost-effectiveness of these emerging sensors combined with their reduced size allows for high density deployments resulting in a higher spatial granularity. In this paper, we briefly present the 3M'Air project that aims to explore the potential of participatory citizen measures using low-cost sensors in order to improve the local knowledge of air quality and temperature and then bridge the gap between individual exposure and regional measurements. We present then the design, implementation and evaluation of our low-cost, small-size WSN-based participatory monitoring system. This system is composed of mobile sensing nodes measuring temperature, humidity and a number of pollutants $\left(\mathbf{N O}_{2}, \mathbf{P M}_{1}\right.$, $\mathbf{P M}_{2.5}$ and $\left.\mathbf{P} \mathbf{M}_{10}\right)$. The collected data are sent to a server for analysis and building temperature and air quality maps. To validate our platform, we have carried out multiple tests to compare our sensor nodes to reference stations and to each other. We have also evaluated the energy consumption of our nodes under different configurations. The results are satisfactory and show that our nodes can be used in environmental participatory monitoring.
\end{abstract}

Index Terms - air quality, urban heat islands, low-cost sensors, participatory sensing, wireless sensor networks, environmental monitoring

\section{INTRODUCTION}

On the one hand, air pollution is one of the main concerns of many cities around the world. Indeed, millions of people live in cities where the concentration of pollutants exceeds the standard health limit several times a year, which constitutes a serious health problem. According to World Health Organization (WHO) data, indoor and outdoor air pollution caused 7 million deaths in 2016 [1]. In fact, long-term exposure to air pollution can result in cancer and damage to the immune, nervous and respiratory systems. Pollution concentration is particularly present in urban cities where the density of population and buildings is higher, augmenting the risks of air pollution exposure [2]. On the other hand, urban heat islands (UHI) greatly increase the risk of allergies, respiratory and cardiovascular problems and can lead to excess mortality among vulnerable people during heatwaves [3]. Moreover, several studies show that the heat island effect contributes to the degradation of air quality by increasing the concentration of some pollutants (ozone, nitrogen oxides, etc.) [4], [5]. Therefore the need to assess air pollution and UHI in our cities is extremely required to establish well adapted public policies.

In order to mitigate the impact of air pollution and UHI, it is essential to set up an effective monitoring system whose main objective is to measure temperature and air pollution concentration and then to produce high resolution maps in real time. Conventional monitoring stations include various sensors, which typically measure a large number of environmental parameters and pollutants, such as temperature, humidity, carbon monoxide (CO), nitrogen oxides (NOx), ozone $\left(\mathrm{O}_{3}\right)$ and particulate matter (PM) [6]. Despite providing accurate concentrations, these monitoring stations are too large and expensive to be deployed anywhere [7]. In addition to traditional monitoring based on measurements, numerical simulations of physical models can also be used to generate maps of air pollution and the UHI. These models simulate the spread of air pollution as a function of the location of pollution sources, land use, emission rates and meteorological data.

The limitations of large monitoring stations in terms of cost, size, flexibility and spatial granularity have led to the emergence of small and low-cost air quality sensors. These sensors have lower accuracy than traditional monitoring stations, but are more flexible and easier to maintain. This would ultimately help improving the spatial and temporal knowledge of air quality and urban heat islands. Compared to traditional air pollution monitoring solutions, the wireless connectivity of low-cost air quality sensors forms a Wireless Sensor Network (WSN), which has many advantages. In fact, their costeffectiveness enables dense, large-scale deployments, which increase the spatial resolution. In addition, both size and cost factors provide greater deployment flexibility. However, low-cost sensors are less accurate than traditional monitoring stations and their data quality is questionable [8]. Indeed, electrochemical sensors present high sensitivity to meteorological changes (temperature, humidity, etc.) and have cross-reactivity problems with similar molecular types. Also, measurements of other low-cost sensor categories such as optical particulate counters may depend on the density, shape of particles or even their color [8].

Mobile crowdsensing is an emerging paradigm that has attracted great attention in the recent years, owing the result of the rapid evolution of Internet of Things (IoT) [9]. This 
paradigm leverages population density to perform large-scale monitoring. One domain of application of mobile crowdsensing among many others is air quality, in which participants use embedded sensors on their smart devices (e.g, smartphones, smartwatches, etc.), or stand-alone sensor nodes to monitor environmental conditions in their surroundings. Therefore, this will result in a better involvement of citizens in the sensing process, measure their individual exposure and giving them access to collaborative maps. In addition, this will help the scientific community to collect more data to improve air quality and UHI knowledge, and the local authorities to implement appropriate policies.

This paper introduces 3M'Air, a project that aims to explore the potential of participatory citizen measures using low-cost sensors in order to improve the local knowledge of air quality and temperature. As part of this project, we present the design, implementation and evaluation of a mobile participatory-based air quality and heat islands monitoring platform based on a network of small sensor nodes carried by people. The remainder of this paper is organized as follows. Section II discusses related research work. Section III presents the context and objectives of this project along with some design guidelines that we have adopted throughout this work. An overview of the proposed system architecture and its main components is presented in Section IV. Subsequently, the obtained experimental results are discussed in section V. Section VI presents briefly some future work and perspectives. Finally, Section VII concludes this paper.

\section{RELATED WORK}

The studies of urban air quality and heat islands in urban areas, in the context of climate change, form today two complementary issues. In fact, the local air quality is impacted by the urban heat island, since it influences both the characteristics of the airflow and the boundary layer of the atmosphere [10]. Hence, the effectiveness of the actions carried out by public spatial planning policies to deal with these two issues is strongly linked to the fine knowledge of temperature gradients and air quality at local scales. To increase the local knowledge of air quality and urban heat islands, many low-cost sensorbased environmental monitoring projects have emerged over the past decade.

A generic environmental monitoring platform based on fixed indoor sensors is presented in [11]. It uses open-source hardware platforms as Arduino and Raspberry Pi, and ZigBee as a communication protocol. The system is composed of sensing nodes, a web interface, and between the two a base station which embeds a gateway node, a database, and a web server. Sampling is performed each $35 \mathrm{~min}$ and measurements are sent using ZigBee to the base station that gathers and stores the data for future visualisation on a web browser using the internet or a local network. The solution has been tested indoor by deploying a base station and 3 sensor nodes measuring both temperature and relative humidity. The authors identify the need of an important number of ZigBee routers for large scale deployments.

An implementation of a flexible environmental monitoring system is described in [12]. The solution is based on a set of small sensor nodes and transceivers communicating in Bluetooth Low Energy (BLE) and a cloud-based back-end. The sensors are equipped with 32-b ARM-M0 micro-controller, a low-power 2.4-GHz transceiver, a 32.768-kHz Real-TimeClock (RTC), a temperature and relative humidity sensor and a local storage that can store up to 125000 measurements. These components are soldered along with a lithium battery on a small Printed Circuit Board (PCB). Measurements are logged at intervals of $15 \mathrm{~min}$ on the nonvolatile memory and sent to a Raspberry Pi Zero W board that pushes data to the cloud. Two possible ways of visualizing data are offered, on a smartphone by connecting it to the node using BLE or on a web interface using a web browser. This solution has been tested indoor in a heritage building in the north of Italy for more than two months. The results point out the challenge of environmental conditions variation from a position to another even at small distances.

Another cloud-based monitoring system is proposed in [13]. The system is composed of a sensor network, a cloud system and an end-user layer. Sensor nodes incorporate a microcontroller, up to four gas sensors measuring a set of volatile organic compounds (VOCs) (benzene, toluene, ethylbenzene, and xylene), a ZigBee module for communicating with the Gateway, and a power management controller that can switch between a battery and a solar panel to power the node. The "ConnectPort X4" Gateway used in this solution integrates a ZigBee module that receives measurement data at the end of each sampling cycle $(10 \mathrm{~min})$ and forwards them to the cloud system where data are stored and processed for delivery to the end-user repsented by an internet application. The solution has been tested in laboratory by generating different compounds at different concentrations using specific tubes containing an analyte that passes through a tube at a maintained speed and temperature. The authors also propose pattern recognition techniques to efficiently detect VOCs and show that they achieve good results in their quantification.

The Citi-Sense-MOB project [14] aims to support green growth and sustainable development through a mobile environmental monitoring system formed by sensor nodes based on electrochemical sensors measuring $\mathrm{NO}_{2}, \mathrm{NO}, \mathrm{CO}, \mathrm{SO}_{2}$, $\mathrm{O}_{3}, \mathrm{CO}_{2}$, temperature, and relative humidity. Two sensing platforms are proposed, one mounted on buses and the other mounted on electrical bicycles. The first platform performs measurements by pumping the air inside a chamber in contact with the sensors and collects position, speed and use of brakes directly from the bus computer and sends the data to a central database. The other platform is powered by the bicycle battery and has no need for a pump as it is in direct contact with the air. Measurements gathering begins as a person starts riding the bicycle and turns off a short period after the bicycle stops. Collected measurements are sent to the same data base as the first platform. Citizens can visualize nodes' measurements and positions gathered from both platforms on a map using a web interface or a mobile app. Through this work, the authors aim at increasing the awareness of citizens and involving them in the adoption of new habits that contribute in the reduction of air pollution.

The Airsense project [15] provides a portable device for 
personal air quality monitoring. The sensor node is batterypowered and integrates the Sharp GP 2Y 1010AU 0F dust sensor that measures particulate matter $\left(\mathrm{PM}_{2.5}\right)$, an SHT15 temperature and relative humidity sensor, an ADXL345 accelerometer sensor to capture users' movements and a GPS module to track users' location. All sensors are incorporated along with a micro-controller on a single PCB. No wireless communication technology is used. Measurements are instead stored on a SD card that is accessible by the user.

City Scanner [16] is a general-purpose portable sensing platform that offers multiple configurations in terms of sensing probes. The solution does not require a specific power supply and therefore can be mounted on top of any urban vehicle without impacting its operations. Each deployed node consists of a group of sensing modules sending their measurements via a short-range WiFi network to a core unit which ensures power management, data storage and streaming to the cloud using open WiFi hotspots. The proposed solution has been deployed in the city of Cambridge. Environmental probes measuring temperature, humidity and particulate matter have been mounted on trash trucks along with other sensors like thermal cameras and WiFi scanners. The main challenges encountered with the deployment were the data transfer reliability, power consumption and data fidelity.

The UrPolSens project [17] proposes a low-cost energyefficient air quality monitoring platform that uses fixed sensor nodes powered by solar panels and batteries. The nodes measure temperature, relative humidity and $\mathrm{NO}_{2}$ and can be adapted to measure PM or VOC. Measurements are sampled every second. Every minute an average is recorded on a shortterm EEPROM. The average measurements are then aggregated into 10-minute records. These records are stored on a local SD card and sent using low-power LoRa communication technology to a gateway that forwards the packets via cellular network to a central cloud server for storage, filtering and processing. A web interface has been developed to visualise real-time air pollution concentrations and weather conditions. The solution was deployed and tested for 3 months in Lyon city in an urban street surrounded by two reference stations. The results show that the system is energy-efficient while keeping an acceptable degree of accuracy.

The OpenSense project [18] studies the performance of low-cost sensing probes by designing and deploying mobile sensor nodes on trams and buses. The nodes measure several environmental parameters such as temperature, humidity, $\mathrm{O}_{3}$, $\mathrm{CO}, \mathrm{NO}_{2}$ and PM. In addition, they are equipped with a GPS and an accelerometer to geolocate the measurements. Each node incorporates a Linux-based core component which stores pollution data locally and streams it to the cloud server using either WiFi or cellular. The platform offers a visualization tool that displays pollution concentrations on top of the map of the region. The project was deployed in Zurich and Lausanne in Switzerland, and mounted on top of 10 trams and 10 buses. The researchers point out the challenge of low-cost sensors calibration and propose techniques to reduce the calibration error.

Table I] summarizes the characteristics of the aforementioned low-cost environmental monitoring platforms: type of deployment (i.e. fixed or mobile), measured environmental parameters, sampling frequency, capacity to store data locally, communication technology, and energy consumption when available.

Temperature and humidity monitoring is present in most of the platforms, proving the importance and the impact of heat islands on other pollutants and air quality in general. Besides, most of the presented platforms use ZigBee, WiFi or BLE which are quite adapted for indoor monitoring or fixedsensors-based monitoring systems. In the context of crowdsensing, BLE is useful to send measurement data to users' smartphones, while sending the data from the smartphones to the cloud needs a cellular connection or WiFi. However, this operation, whether it requires a human intervention or not, consumes a significant amount of the smartphone's energy and mobile data plans. As an alternative, low-power long-range communication technologies could cover the whole network of sensors with a small number of gateways.

\section{3M'AIR PROJET}

3M'Air ("Mobile Citizen Measurements and Modeling: Air Quality and Urban Heat Islands") is a three-year multidisciplinary project that aims to explore the potential of participatory sensing to improve the local knowledge of air quality and Urban Heat Islands. It leverages several scientific expertises going from electrical engineering, computer science and fluid mechanics to geography, urban climatology and sociology.

\section{A. Context and Objectives}

The adverse effect of urban heat islands and air pollution on human health and the environment has been widely documented in several studies [19], [20], [21]. Despite that, pollutant concentrations as well as air temperature continue to increase, mainly due to urbanization, industrialization and other factors [22] [23] . For instance, in Lyon city, France, an increase of $2.2{ }^{\circ} \mathrm{C}$, representing $+0.4{ }^{\circ} \mathrm{C} /$ decade, was recorded in average annual temperatures between 1959 and 2016 [24] [25]. More precisely, during summer, there is an increase of 2.8 ${ }^{\circ} \mathrm{C}$ between 1959 and $2016\left(+0.5^{\circ} \mathrm{C} /\right.$ decade $)$ [24]. In order to i) understand the link between microclimates and the potential impact of UHI and urban pollution, and to ii) bridge the gap between individual exposure and regional measurements, a fine characterization of temperature and air quality at local scales is needed. Our project aims to evaluate the added value of moderately accurate pollution data generated by a nonscientific community in the fine-grained characterization of air quality and urban heat islands. Our project also seeks to involve citizens in the scientific process of air quality monitoring and to present adapted and optimized approaches for participatory data analysis to generate fine-grained pollution maps while taking into account the continuity in space and time of measurements and the dynamic nature of the studied phenomena. We believe that the interest of these participatory data will be stronger by combining them with physical models and accurate reference monitoring stations. To this end, the first step of our project is to propose a multi-application participatory monitoring system with a variety of measured 


\begin{tabular}{|c|c|c|c|c|c|c|}
\hline Projects & Fixed / mobile & Measured parameters & Sampling frequency & Local storage & $\begin{array}{c}\text { Communication } \\
\text { technology }\end{array}$ & Energy consumption \\
\hline Ferdoush and Li [11] & Fixed & $\begin{array}{l}\text { Temperature and } \\
\text { relative humidity }\end{array}$ & $35 \mathrm{~min}$ & No & ZigBee & I \\
\hline Lombardo et al. [12] & Fixed & $\begin{array}{l}\text { Temperature and } \\
\text { relative humidity }\end{array}$ & $15 \mathrm{~min}$ & Yes & BLE & $20 \mathrm{~mA}$ \\
\hline Arroyo et al. [13] & Fixed & VOCs & $10 \mathrm{~min}$ & No & ZigBee & 104 to $270 \mathrm{~mA}$ \\
\hline Castell et al. [14] & Mobile & $\begin{array}{l}\mathrm{NO}_{2}, \mathrm{NO}, \mathrm{CO}, \mathrm{SO}_{2}, \\
\mathrm{O}_{3}, \mathrm{CO}_{2} \text {, temperature } \\
\text { and relative humidity }\end{array}$ & $30 \mathrm{sec}$ & No & Bluetooth & 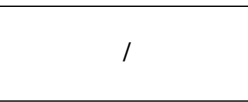 \\
\hline Airsense [15 & Mobile & $\begin{array}{l}\mathrm{PM}_{2.5}, \text { temperature and } \\
\text { relative humidity }\end{array}$ & $3 \mathrm{sec}$ & Yes & I & I \\
\hline City Scanner [16] & Mobile & $\begin{array}{l}\text { Temperature, humidity } \\
\text { and particulate matter }\end{array}$ & l & Yes & WiFi & l \\
\hline UrPolSens [17] & Fixed & $\begin{array}{l}\text { Temperature, humidity } \\
\text { and } \mathrm{NO}_{2}\end{array}$ & $1 \mathrm{sec}$ & Yes & LoRa / Cellular & $18 \mathrm{~mA}$ \\
\hline OpenSense [18] & Mobile & $\begin{array}{l}\text { Temperature, humidity, } \\
\mathrm{O}_{3}, \mathrm{CO}, \mathrm{NO}_{2} \\
\text { and particulate matter }\end{array}$ & I & Yes & WiFi / Cellular & Permanently plugged \\
\hline
\end{tabular}

COMPARISON OF DIFFERENT ENVIRONMENTAL MONITORING PLATFORMS

parameters (gas, particulate matter, temperature and relative humidity) based on lab-designed modular, autonomous and optimized sensor nodes whose hardware and software can be adapted to the needs of our studies.

\section{B. System Design guidelines}

Based on the study and survey results, our first aim was to design a generic network architecture that mainly serves participatory environmental monitoring applications but can be easily adapted to other environmental applications. To achieve this goal a number of recommendations need to be respected. Our main design guidelines can be summarized as follows:

Required parameters: In order to serve the two identified applications (i.e. air quality and urban heat islands), the designed node should measure air temperature, relative humidity, $\mathrm{NO}_{2}$, $\mathrm{PM}_{1}, \mathrm{PM}_{2.5}$ and $\mathrm{PM}_{10}$. Note that an extra calibration is often needed to extract meaningful data from sensors like conversion from voltage to pollutant concentration for gases like $\mathrm{NO}_{2}$, or conversion from particle counts to mass for particulate matter.

Data gathering: The network should allow measuring and gathering all the identified air and weather parameters at a remote server in order to allow the remote monitoring via internet. Moreover, to offer a good data availability, it is preferable to store the collected data at multiple levels. This will add robustness against network connection problems.

Autonomy and network lifetime: The sensor nodes are designed to be carried by people, hence they should deliver at least 12 hours of operating time. This does not just mean integrating a bigger battery but also optimizing all the system components to offer better efficiency.

Sampling frequency: The developed network must be able to perform measurements at an acceptable sampling rate to ensure a good spatio-temporal coverage. The system must be easily configurable to be adapted to the mobility of users (e.g. bicycles or vehicles,...). However the main priority is the use on foot, meaning an average speed between and 0.83 and 1.57 $\mathrm{m} / \mathrm{s}$ [26], [27], [28]. By setting the default sampling frequency to one every $20 \mathrm{sec}$, consecutive readings are less than $30 \mathrm{~m}$ apart, which gives a good spatio-temporal resolution.
Modular design: Our objective is to design a platform that will be as flexible and extensible as possible. This can be achieved by opting for a component-based design where the whole system is composed of multiple hardware or software components that can be removed or extended when needed.

Ease of use: Internet of things involves a big number of smart connected devices in our daily lives. This should not complicate our life but on the contrary simplify it. For this reason, the designed nodes need to be light and small. Even more, they should not require technical or scientific knowledge to be used. It should be simple and as intuitive as possible to turn on the node, to use it and to charge it.

Reliable measurements: To guarantee a good data reliability, the sensor nodes should be adapted to real environmental conditions. In fact, solar radiations have a big impact on temperature and humidity measurements but also on other pollutant measurements. To protect the sensor probes from the effect of solar radiations, the nodes should be white and incorporate a solar radiation shield.

\section{SYSTEM ARCHITECTURE}

The sensing layer is composed of low-cost, small-size, battery-powered and portable wireless nodes incorporating three environmental sensors: a dust sensor (measuring $\mathrm{PM}_{1}$, $\mathrm{PM}_{2.5}$ and $\mathrm{PM}_{10}$ ), a $\mathrm{NO}_{2}$ sensor, and a temperature/humidity sensor. In addition, the nodes include a power manager, a GPS receiver, a microSD card module (to ensure data availability in case the node is outside LoRaWAN network coverage), an analog-to-digital converter (ADC) to enable compatibility with analog sensors, and a LoRa module. All the peripheral components are managed and orchestrated by a micro-controller.

The communication layer relies on the LoRaWAN infrastructure provided by "The Things Network" (TTN) [29]. LoRaWAN [30] is a networking protocol defined by the LoRa Alliance which is an organization of more than 500 companies collaborating to promote the LoRaWAN open standard. This standard is designed for sending small data packets over long distance at a low bit rate and is one of the most promising Low-Power Wide-Area Network (LPWAN) technologies for 


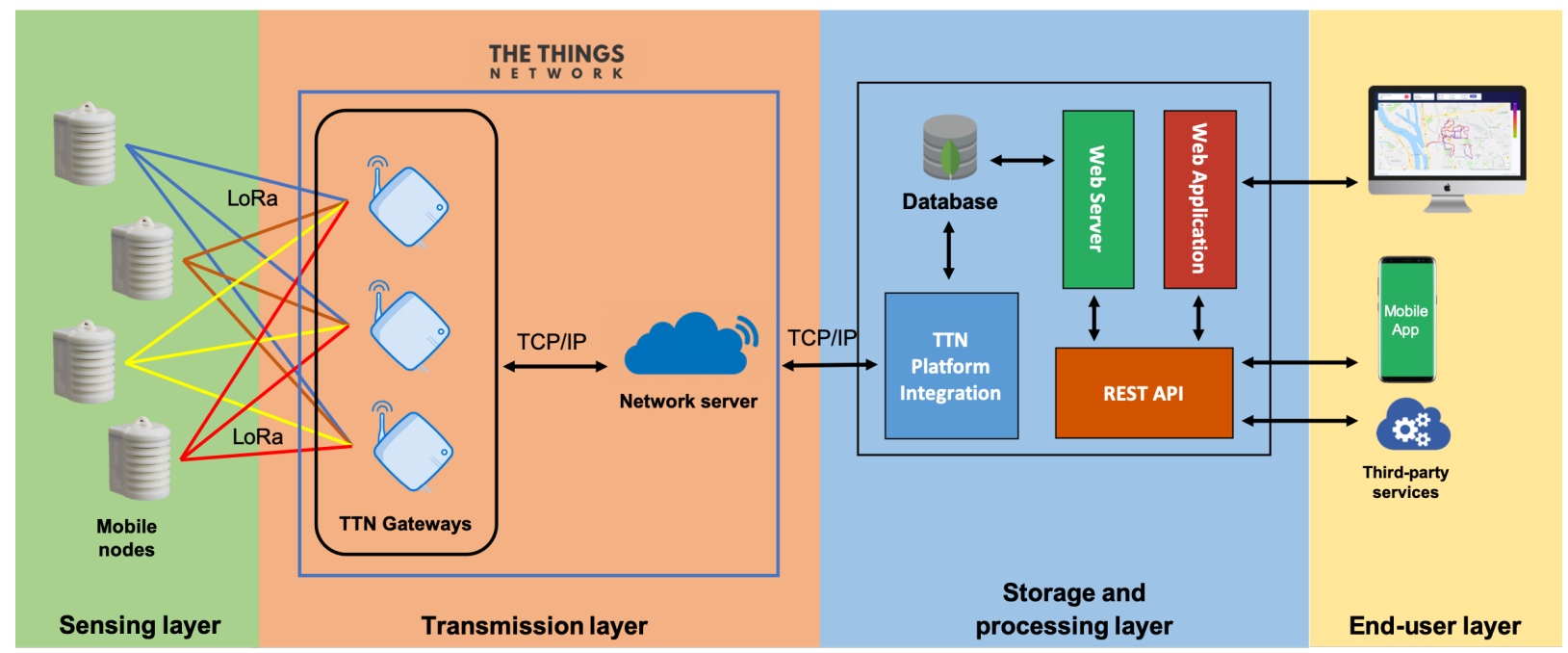

Fig. 1. System architecture overview

Internet of Things [31] . LoRaWAN relies on LoRa physical layer which uses Chirp Spread Spectrum (CSS) as modulation and offers multiple orthogonal Spreading Factors (SF).

LoRaWAN offers the possibility of using eight configurations (Data Rates) on the EU863-870MHz ISM Band with six possible SF values (SF7 to 12) [32]. The six mandatory configurations use a $125 \mathrm{kHz}$ bandwidth and offer a bit rate varying from $250 \mathrm{bps}$ for SF12 to $5.47 \mathrm{Kbps}$ for SF7. Two more configurations use respectively a $250 \mathrm{kHz}$ bandwidth with SF7 achieving a bit rate of $11 \mathrm{Kbps}$, and FSK modulation offering $50 \mathrm{Kbps}$ [32]. We note that the LoRaWAN maximum payload size ranges from 51 to 222 bytes depending on the selected $\mathrm{SF}$ value [32].

However, LoRaWAN has restrictions due the used frequency band. For instance, in Europe, most of the $868 \mathrm{MHz}$ sub-bands have a duty cycle of $1 \%$, thus, each node must not exceed $1 \%$ of spectrum occupancy per channel and per SF.

In addition to the physical configuration, LoRaWAN also defines the network architecture as well as the upper networking layers.

In the proposed architecture by LoRaWAN, the gateways forward the packets sent by sensor nodes to the network server, which does the filtering of the data and makes sure that only one copy of the packet is sent to our application server to avoid data redundancy.

On top of that, LoRaWAN offers two security modes: i) Over The Air Activation (OTTA) mode where a join procedure is performed at the beginning in order to generate dynamic addresses and security keys, and ii) Activation By Personalization (ABP) mode where addresses and security keys are pre-loaded before the deployment. In both modes, a Message Integrity Code (MIC) is added to each message to ensure that data has not been changed and is used by nodes and the network server to ensure data integrity. Thanks to the payload encryption, confidentiality is also ensured between nodes and the application server in both modes. We choose to use OTTA as it is based on dynamic keys, and handles the initial nodes authentication in addition to the confidentiality and data integrity.

TTN is a contributor member of the LoRa Alliance offering a free-to-use LoRaWAN network. They manage the cloud infrastructure while the gateways are mainly deployed by volunteers (including our lab) [33]. This layer is responsible for forwarding measurement data received from the nodes to the cloud server which represents the third layer. We have selected this solution for mainly three reasons: 1) the participatory aspect of our platform that meets the collaborative nature of TTN; 2) its robustness and security through a large number of gateways and an end-to-end encryption; 3) its growing adoption in many fields all over the world with an increasing number of deployed gateways [34], [35], [36].

The storage and processing layer is implemented on a multiple functions cloud server. It receives data from the TTN backend and stores them into a NoSQL database for cleaning (i.e. correcting or removing inaccurate or redundant data from the database) and processing. It offers a REST API through which measurement data can be queried using a web browser, a mobile application or a third-party service.

The end-user layer offers the users the possibility to visualise air pollution concentrations and weather parameters using either a mobile application, a third-party service, or a web interface that we designed to display maps and statistics.

\section{A. Sensing and transmission}

Our aim in this project is to design small and portable nodes based on low-cost sensors. Given the fact that these nodes are designed to be mobile and carried by people, it is important to have a suitable design to 1) ensure the node's functioning in mobility, 2) protect the node from solar effects while guaranteeing air flow, 3) maintain a reliable communication, and 4) make the nodes the lightest possible for users. We have designed a casing with an integrated solar radiation shield and three separated chambers as depicted in Figure 2. We modeled the casing using Autodesk Fusion 360 software. The casing was 3D-printed using a Selective Laser Sintering process with Nylon PA12 plastic. This material provides good strength and 
enough flexibility to withstand a fall without damaging the node. The $16 \mu \mathrm{m}$ print resolution allows precise placement of the components as well as their attachment directly to the casing.

The first chamber regroups the environmental sensors and is designed to provide natural ventilation while protecting the sensing probes from solar radiations. For the sensors, we employ the AlphaSense series $B$ [37] sensor which measures $\mathrm{NO}_{2}$ concentrations. This low-cost electrochemical probe produces a current from the interaction of the target gas with the electrolyte [38]. The Grove HM3301 low-power laser dust detection sensor measures three sizes of particulate matters $\left(\mathrm{PM}_{1}, \mathrm{PM}_{2.5}\right.$ and $\left.\mathrm{PM}_{10}\right)$. This sensor is equipped with a fan driving airflow inside a detection chamber, and is based on laser light scattering technology. It has an effective detection range of 1 to $500 \mu \mathrm{g} / \mathrm{m}^{3}$ [39]. The last sensor is the $D H T$ 22 , a small low-power sensor based on a polymer capacitor for measuring temperature and relative humidity with an operating range of -40 to $80{ }^{\circ} \mathrm{C}$ and 0 to $100 \%$ respectively [40].

The second chamber holds a printed circuit board (PCB) that we designed to integrate all necessary components. Our PCB incorporates an Arduino MKR WAN1300 built on the Atmel SAMD21 low-power ARM micro-controller [41], the Murata CMWX1ZZABZ LoRa module $32 \mathrm{~KB}$ SRAM [42], 8 digital pins, and 7 Analog input pins as depicted in Figure 3 An $M K R$ $M E M$ ASX00008 shield is used to add a microSD card port and $2 \mathrm{MB}$ extra flash memory. For managing analog sensors, a 16bits ADS1115 analog digital converter (ADC) [43] has been added to have a better precision than the embedded MKR 12-bits ADC. Geolocation is achieved by a low-power GPS receiver based on the MTK3339 chipset. This high-sensitivity module has 66 channels and can track up to 22 simultaneous channels [44]. In addition, the GPS module has a small battery allowing it to save the current date and time without having to perform a data acquisition from satellites. This is very helpful in situations where no satellite coverage is available (e.g. passing into a building or going inside a metro station). The last component on the PCB is the PowerBoost 1000C, a small power manager [45] based on a DC/DC boost converter chip that powers the system and is connected to the $3.7 \mathrm{~V} \mathrm{LiPo}$ battery placed in the third chamber. It is possible to charge the node's battery by plugging the node to an external $2 \mathrm{~A}$ power source using an easily accessible micro USB port located at the bottom of the node. An additional micro USB port is also available for node programming and debugging purposes.

When the node is turned on, the micro-controller initializes the different modules and establishes an Over The Air Activation connection to our application on the TTN network. Data are gathered from all environmental sensors and the GPS at 20 seconds intervals. In order to reduce the energy consumption of data transmission and storage while respecting the maximum payload of LoRaWAN packets, each node accomplishes three measuring cycles before storing the data on the micro-SD card and sending them to TTN gateways. The data record contains the ID of the sensor, the timestamp of the measurement, temperature, relative humidity, $\mathrm{NO}_{2} \mathrm{PM}_{1}$, $\mathrm{PM}_{2.5}, \mathrm{PM}_{10}$ values and GPS coordinates. In case there is no GPS signal, the GPS coordinates will have the value zero, but we still can get the exact date and time from the GPS, thanks to the GPS module's battery that allows it to save the current date and time.

We have formatted our packets to contain 51 bytes (3 $\mathrm{x} 17$ bytes) which satisfies the duty cycle constraint using SF7, SF8 and SF9 with a 20-second sensing period and one-minute transmission period. We note that the value (3) is the number of cycles and is also configurable. We also note that higher values of SF can be used by increasing the transmissions period and/or reducing the amount of data to send (e.g. compressing data, reducing the measurement precision, sending averaged data).

In all our tests we send confirmed packets (i.e. the application server has to acknowledge the reception of the packet). However, we do not implement retransmissions since data are stored locally on the nodes and the loss rate was very low. Indeed, thanks to the duty cycle, the collision probability is low when the number of nodes in a close proximity is not large [46].

When packets are received by $\mathrm{TTN}$, they are forwarded to a router/broker service in the TTN infrastructure that will decode the message. The latter is then published to the right application handler which is our cloud server. For further details on how the transmission layer works, please refer to the TTN documentation [29].

\section{B. Storage and processing cloud server}

The large data volume generated by the sensor nodes needs to be stored in a remote server in addition to the local storage. Hence, providing a new layer of storage to ensure the availability of the data and offering the possibility to access data remotely. In order to catch the data sent by the transmission layer we developed a Node.js script based on the integration solution provided by TTN which offers a set of open tools to facilitate the development of IoT applications. Whenever a new measurement is received from the gateway, it is parsed and then stored on a NoSQL database (MongoDB) using Mongoose which is an object modeling tool that provides schema-based solution to model application data.

The cloud server is composed of two main components; the database component and the end-user services component. The latter is responsible of answering user requests through a REST API for mobile apps, third-party services, or a web interface which is accessible via any desktop or mobile web browser as shown in Figure 1. In addition to that, multiple data processing techniques can be added such as detection of outliers and redundant data, measurements calibration, concentrations prediction, missing data reconstruction, etc.

\section{End-user application description}

In order to visualize the sensor nodes measurements, we have developed a web application that offers a simple and intuitive interface for visualizing pollutant concentrations and weather parameters. The front-end of the web application is built using HTML, CSS and Angular which is an open-source component-based front-end framework for building large-scale single-page applications. It is built on TypeScript which relies 

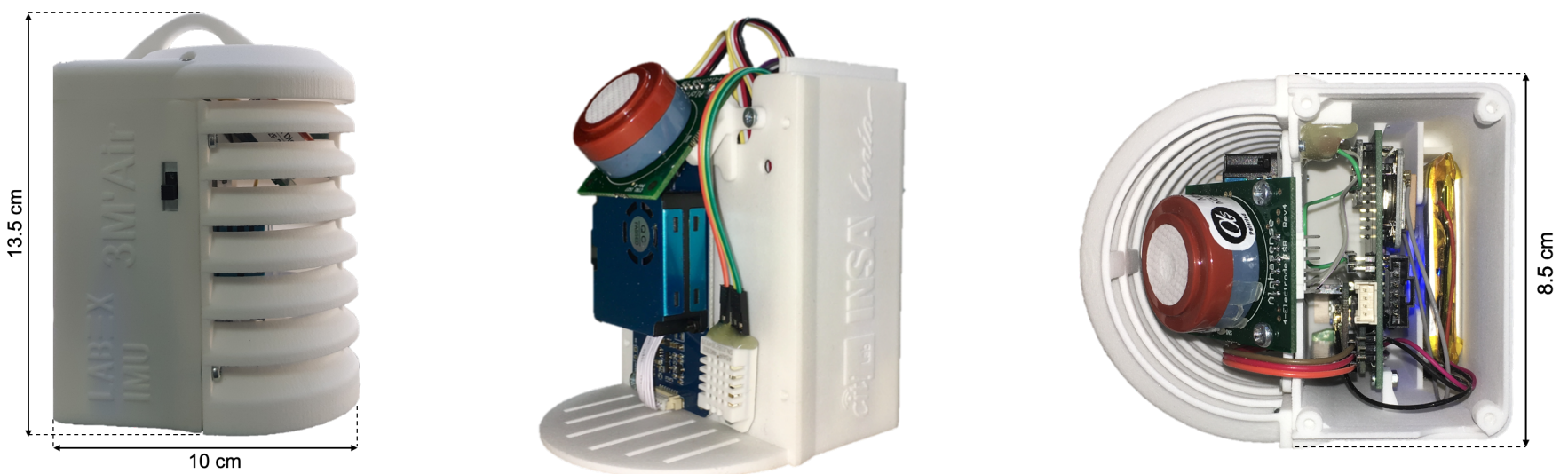

Fig. 2. (a) External side view of the designed sensor node; (b) Internal side view (sensor chamber); (c) Internal top view (second and third chambers)

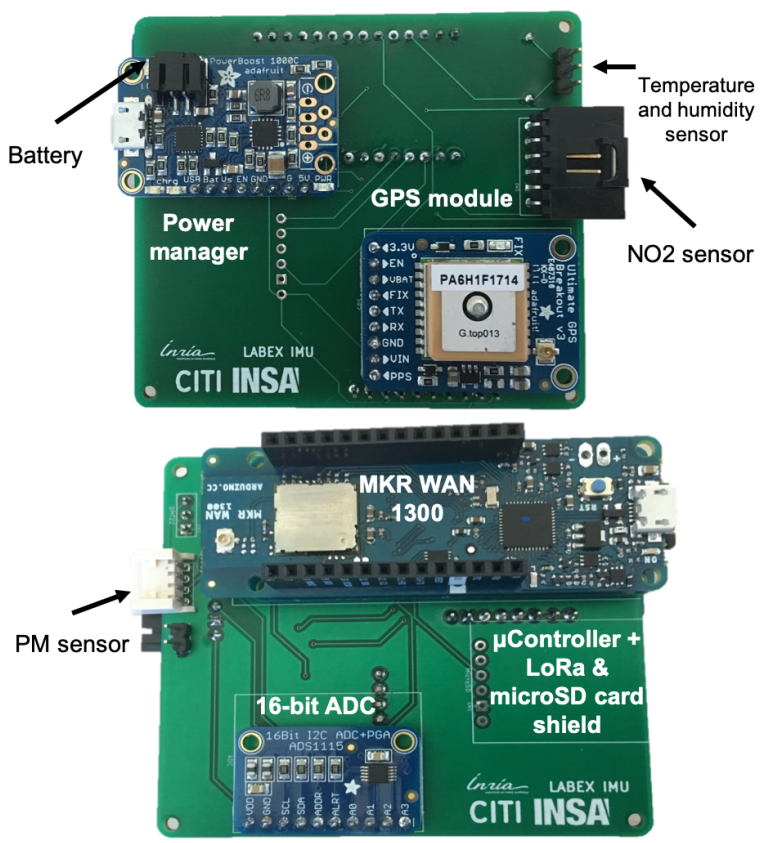

Fig. 3. The node PCB with the different modules embedded

on JavaScript ECMAScript6 and offers a lot of features that simplify web development such as dependency injection and component independence. The interface is based on Bootstrap 4 and Angular Material 7 for good looking UI components that work across the desktop and mobile.

For experimentation purposes, we have designed two frontend components: Dashboard and pollution maps as depicted in Fig. 4 and Fig. 5 Through the first component, it is possible to display useful information such as the list of nodes along with their IDs, LoRaWAN addresses and last time seen online, etc. The dashboard also presents statistics about the contribution of each node to the application as well as the total number of measurements or a the number of measurements performed per month. All the information presented in the dashboard is actively updated in real-time to keep the user informed about

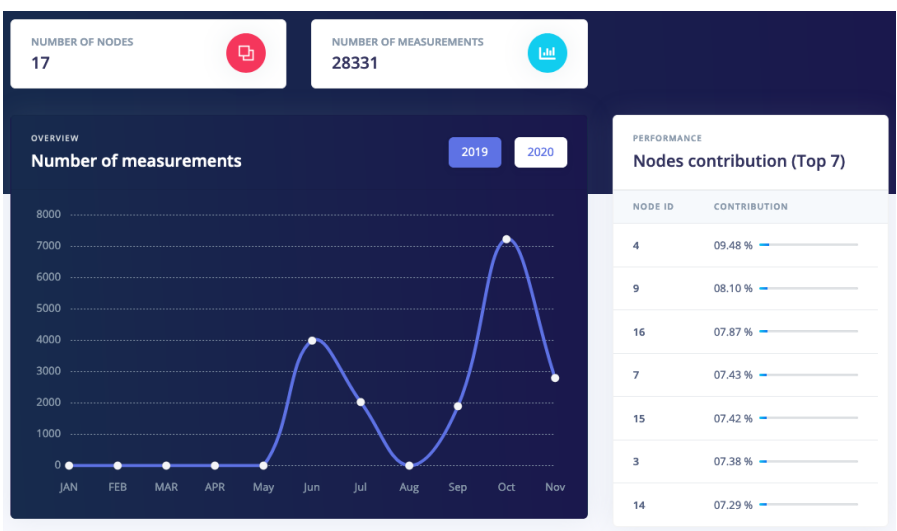

Fig. 4. The web dashboard of the platform

the last changes. The Maps component allows the user to visualize air quality and meteorological conditions within the city by choosing the wanted date and parameter to visualize. All requests generated from the web application are sent to the web server asynchronously to improve the user experience with non blocking data loading and waiting times.

\section{RESULTS AND DISCUSSION}

Following the aforementioned guidelines and architecture, we have built 16 mobile sensor nodes. Although, before using them in measurement campaigns, we have tested the proper functioning of our nodes by: 1) comparing the sensor nodes to reference stations; 2) comparing the sensor nodes to each other; 3) evaluating their performance in terms of energy consumption. For this, we performed multiple tests which we summarize in what follows.

\section{A. Measurements validity: comparison with reference stations}

To assess the accuracy of our nodes' measurements and the effect of environmental conditions, we have tested them next to two reference devices: the first one is the "Météo France" 


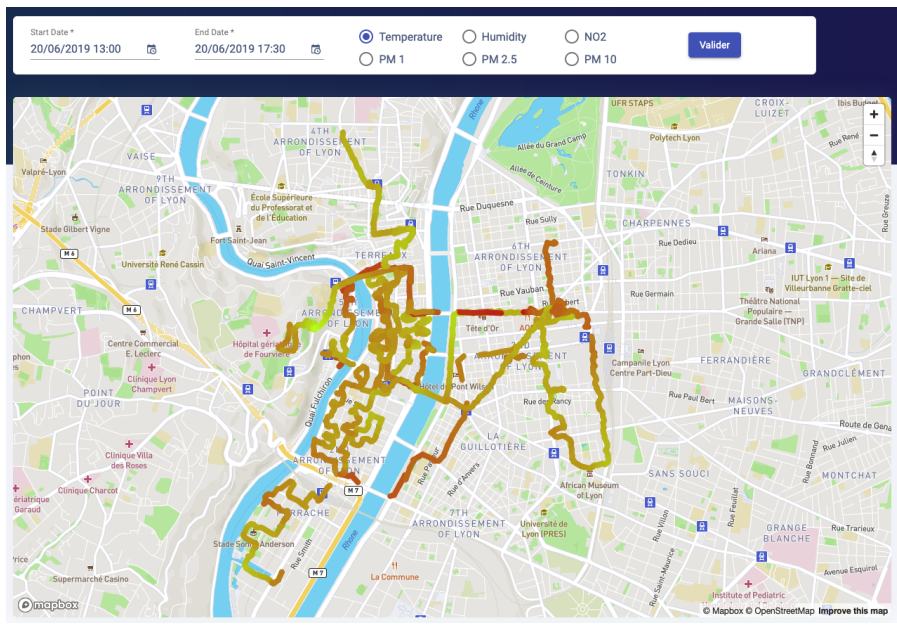

Fig. 5. The web interface showing the measurements of the nodes on a map

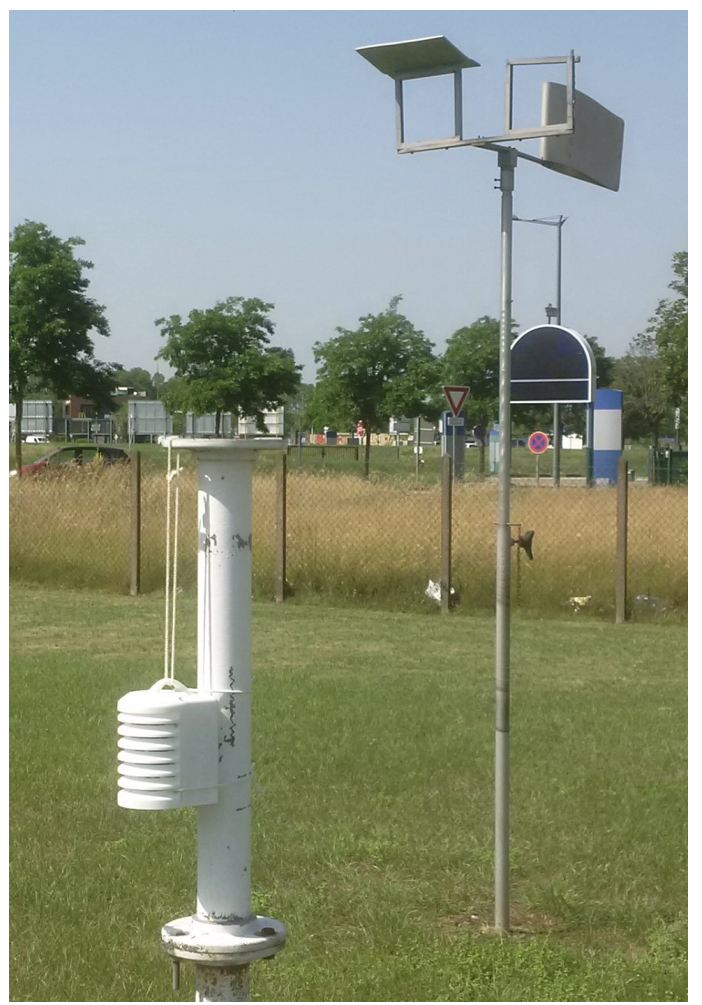

Fig. 6. Our deployed node next to "Météo-France" temperature and relative humidity monitoring station

temperature and relative humidity monitoring station in Lyon city, and the second one is an approved fine dust measurement device (Fidas $200^{\odot}$ ).

1) Temperature and relative humidity: In order to validate the temperature and relative humidity measurements taken by the DHT22 sensor, a metrological test was carried out during summer of 2019, from June 27th at 11am to June 28th at 10am (local time). This date was selected for the high temperatures recorded this day, during the heat wave of late June in Lyon, with a temperature that reached 38.10 ${ }^{\circ} \mathrm{C}$ at $5 \mathrm{pm}$. The 3M'Air measurement device was placed on the instrumentation field of "Météo-France" in Lyon, on a 1 meter high platform (see Figure 6). The measurement site meets the standards recommended by the World Meteorological Organization (WMO) for temperature and relative humidity measurements. The reference sensors of the station are the PT100 for temperature and Vaisala HMP110 for relative humidity. For this test, two samples of measurements have been prepared to evaluate the capabilities of the device measurement as a function of direct solar radiation. Thus, the first sample regroups measurements with direct solar radiation taken from 11am to 9:30pm (sunset at 9:34pm) and from 6am to 10am (sunrise at 5:53am). The second sample includes measurements without direct solar radiation performed from $10 \mathrm{pm}$ to 5:30am. Measurements from both devices are then compared next to each other. Since the data do not follow a normal distribution, the non-parametric Mann-Whitney test is used here (also called Wilcoxon-Mann-Whitney or Wilcoxon Rank-Sum test) [47], [48]. The classical linear regression parameters are also used to compare the performance of the two sensors (R, RMSE and bias). Temperature and relative humidity measurements from both sensors are plotted (see Figure 7).

The test indicates a significant difference $(\mathrm{p}$-value $=0.021)$ in daytime temperatures with 3M'Air recorded values (average temperature of $34.8{ }^{\circ} \mathrm{C}$ ) higher than the reference sensors measurements (average of $32.7{ }^{\circ} \mathrm{C}$ ). Significant differences are recorded punctually, with for example a difference of $4.30{ }^{\circ} \mathrm{C}$ at $11 \mathrm{am}$ and $5{ }^{\circ} \mathrm{C}$ at $8 \mathrm{am}$. The correlation coefficient is 0.93 , The RMSE is $1.7{ }^{\circ} \mathrm{C}$ and the bias is equal to $1.9{ }^{\circ} \mathrm{C}$. On the other hand, no difference is detected for nocturnal temperatures ( $\mathrm{p}$-value $=0.451, \mathrm{R}=0.99$, RMSE $=0.4$, bias $=-0.5)$. No difference was detected in relative humidity measurements either, neither for the day data ( $\mathrm{p}$ value $=0.720, \mathrm{R}=0.97, \mathrm{RMSE}=4.3$, bias $=1.4$ ), nor for the night data ( $\mathrm{p}$-value $=1, \mathrm{R}=0.99, \mathrm{RMSE}=1.4$, bias $=0.1$ ).

The differences obtained for the daytime air temperatures was expected because of the overheating of the shelter with direct exposure to solar radiation. The correlation coefficient for the temperature difference between the two sensors and the global radiation measured at the weather station is equal to 0.53 . Indeed, although our node has an anti-radiation shelter, it is only open on $180^{\circ}$ for design reasons (to keep the node small, the different chambers of the node were placed one behind the other as depicted in Figure 2). In addition, the small size of the sensor node may play a role in this. The measurements of humidity and night temperature are, for their part, totally satisfactory. It is worth mentioning that the 3M'Air sensor nodes were not designed to perform static but mobile measurements.

To evaluate the node's performance in mobility, six tests in four days during the period of July-September have been performed with the 3M'Air sensor node next to two sensor nodes incorporating the $\log 32$ sensor [49] inside two types of anti solar radiation shelters (TFA [50] and DAVIS [51]). In two tests, all sensors were carried by participants walking on foot using straps, and in the last ones, the sensors were placed in a bicycle basket. All tests took place in the "Presqu'île" peninsula of Lyon city and measurements were taken every 


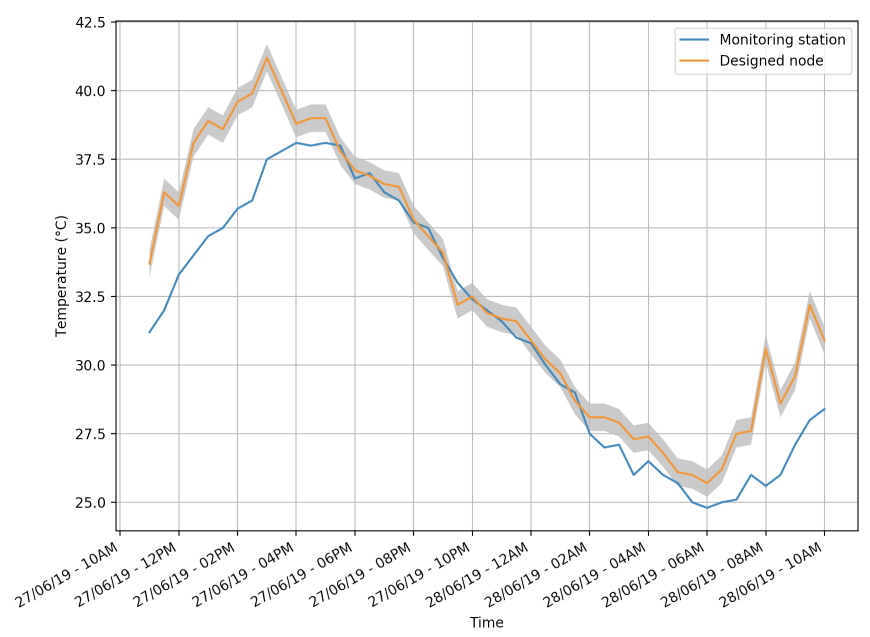

Fig. 7. 3M'Air node vs reference station (a) temperature; (b) relative humidity

20 seconds. Three non-parametric statistical tests have been used on the data gathered by the sensors; Mann-Whitney, Komogorov-Smirnoff [52], Kruskal-Wallis [53].

The results showed divergence between the tests outputs. Indeed this difference is due to the fact that unlike the 3M'air anti solar radiation shield, the TFA and DAVIS shelters are opened on 360 degrees allowing air flow in all directions. In addition both sensor nodes with the TFA and DAVIS shelters are much bigger than our sensor node and do not include a circuit with an important number of components.

We have also calculated the classical linear regression parameters (R, RMSE, and bias). For temperature measurement, the highest coefficient of determination we have got is equal to 0.95 and the lowest is equal 0.61 with an average of 0.82 . The root mean square error is between 0.4193 and 0.1631 degrees Celsius and the measurement bias equals $0.37^{\circ} \mathrm{C}$. For relative humidity, The RMSE belongs to the interval [0.70 and 1.46]\% while the coefficient of determination varies between 0.58 and 0.94 . The measurement biais is around $0.93 \%$. These results show clearly that we are within the error ranges indicated for the DHT22 sensor [40] which make the use of 3M'Air nodes very satisfactory in mobile measurements.

2) Particulate matters: We have evaluated the performance of the Grove HM3301 PM sensor embedded in our sensor node by testing it next to an approved fine dust measurement device called FIDAS $200^{\odot}$ which is TUV Rheinland certified, and recognized by the LCSQA ("Laboratoire Central de Surveillance de la Qualité de l'Air") for monitoring particulate matter concentrations. The two sensor nodes were placed indoor one next to the other from October 4th,2019 to October 9th,2019. The series of measurement are highly correlated with a low RMSE for $\mathrm{PM}_{1}$ and $\mathrm{PM}_{2.5}$ while performances degrades for PM10 as reported in Table $\Pi$. This may indicate that the composition of the largest PM in our region is different from that assumed when the sensor was calibrated. Another interesting fact is that the 3M'Air sensor slightly overestimates the measurements in comparison to the reference device as shown for $\mathrm{PM}_{2.5}$ in Figure 8 Considering the high

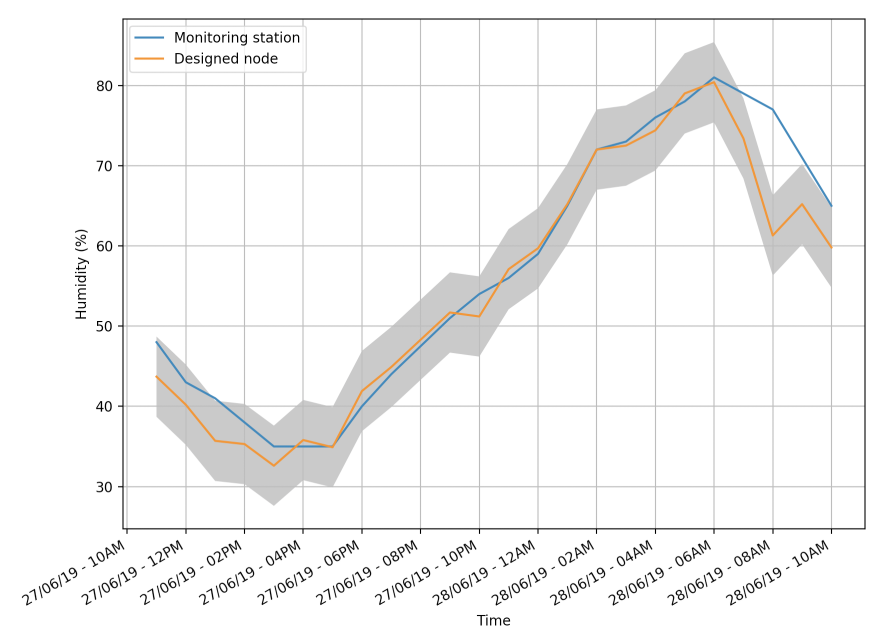

\begin{tabular}{|c|c|c|c|}
\hline Metric & $\mathrm{PM}_{1}$ & $\mathrm{PM}_{2.5}$ & $\mathrm{PM}_{10}$ \\
\hline RMSE & $3.86\left(\mu \mathrm{g} / \mathrm{m}^{3}\right)$ & $5.784\left(\mu \mathrm{g} / \mathrm{m}^{3}\right)$ & $7.92\left(\mu \mathrm{g} / \mathrm{m}^{3}\right)$ \\
\hline $\begin{array}{c}\text { Pearson Correlation } \\
\text { coefficient }\end{array}$ & 0.98 & 0.92 & 0.63 \\
\hline
\end{tabular}

TABLE II

RMSE AND CORRELATION COEFFICIENT OF $\mathrm{PM}_{1}, \mathrm{PM}_{2.5}$ AND $\mathrm{PM}_{10}$ MEASUREMENTS TAKEN BY THE DESIGNED NODE NEXT TO A REFERENCE DEVICE

correlation, the over-estimation can be compensated by an offline correction on the calibration function.

Sensor calibration is needed to cope with the low accuracy and signal drifting of low-cost sensors. In fact, calibration techniques for low-cost sensors have been extensively discussed in the literature. They are generally classified according to the availability of reference stations (reference-based, blind, or partially blind calibration), mobility of the sensors (static, mobile, or hybrid), calibration relationship (univariate or multivariate), and the used calibration model (e.g ordinary Least Squares, multiple Least Squares etc.). [54], [55]

As a proof of concept, we have implemented a univariate linear regression to calibrate our PM sensor's raw data based on the concentrations observed by the FIDAS sensor. In this process, we vary the learning duration and the temporal resolution to evaluate their impact on the calibration performance. Results show that with 6 hours of training on data with 1minute temporal resolution, the RMSE decreases from 5.785 to 0.835 as can be observed on Figure 9 A training duration of 12 hours with the same temporal resolution allows to achieve a smaller error of 0.734. Moreover, when decreasing the temporal resolution to 1 hour instead of 1 minute, the calibration achieves better performance with a RMSE decreasing from 5.735 to 0.558 using 6 hours of training and 0.465 with 12 hours of training. These observations show that high temporal resolution allows to obtain better results. Interested reader can refer to [54], [55] for more details on low cost sensor calibration techniques. 


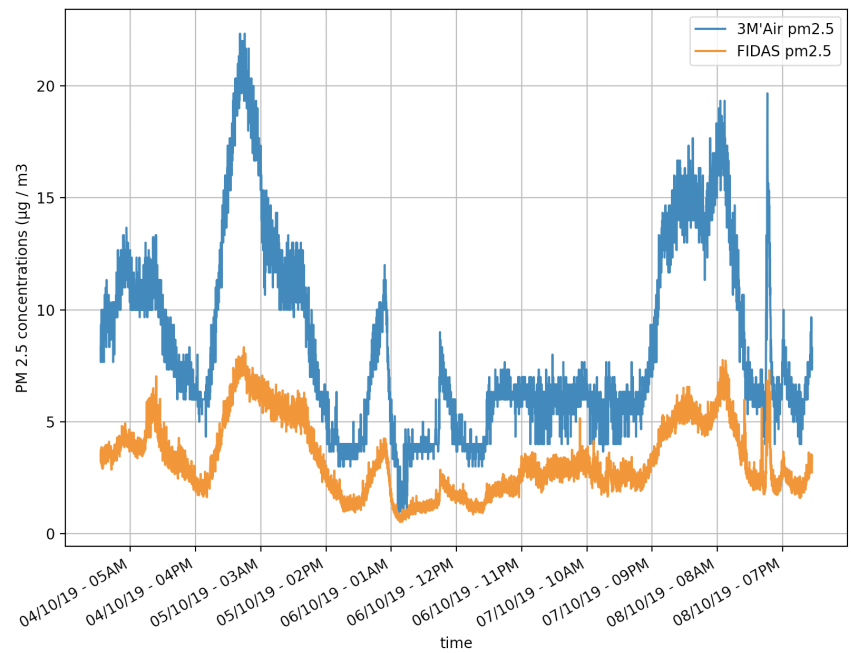

Fig. 8. 3M'Air node vs reference station: $\mathrm{PM}_{2.5}$ concentrations

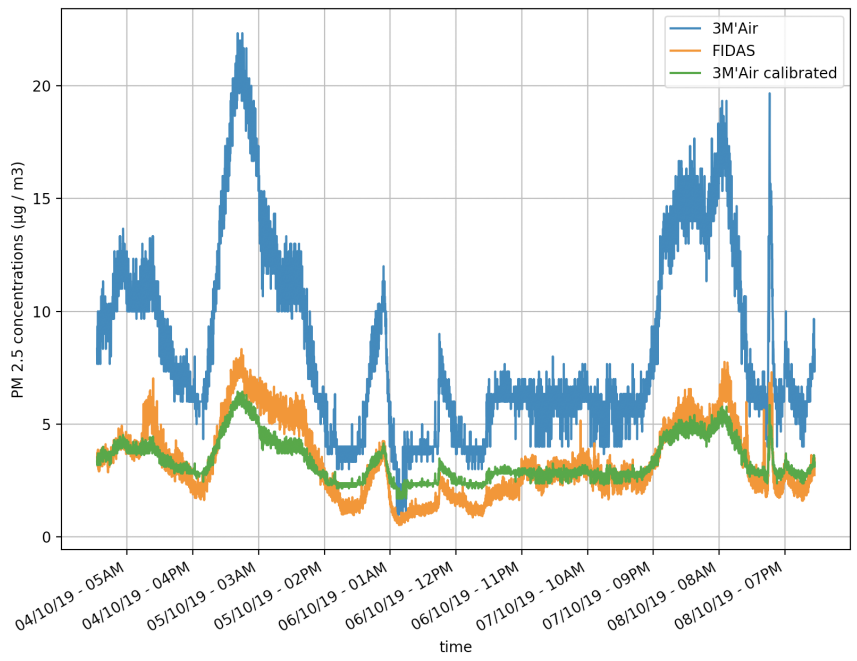

Fig. 9. calibration of 3M'Air PM sensor based on reference station

\section{B. Inter-comparison}

We have also conducted other tests to compare the designed sensor nodes to each others. For this purpose, six nodes forming three groups (two nodes per group) have been deployed on the rooftop of a three-story building. Each group of sensors has been placed differently.

The reason behind this is to evaluate the impact of nodes' position and orientation. The test has been performed from January 21 st,2020 at $12 \mathrm{pm}$ to January $22 \mathrm{nd}, 2020$ at $8 \mathrm{am}$ (local time). While aggregating data into one minute averages, we have calculated the RMSE and the correlation coefficient of Pearson within each group and also between averaged data of the three groups. Results are presented in Table III

1) Temperature and relative humidity: We have compared temperature and relative humidity measurements of every two sensors of each group. Figure 10 presents temperature measurements from all six sensors. The first observation that can be drawn from this figure is that each group measured different temperature and relative humidity values. This was expected since the groups were placed in different positions and orientations, resulting in different sun exposure and wind direction. In addition, there is a good correlation between the nodes of the same group especially with temperature measurements. We also notice that the difference in measurements between the groups still existed despite the absence sunrays. This confirms that measurements can be impacted not only by the sun exposure but also by the wind direction. Another observation that can be noted from Table $\amalg$ is that the RMSE of temperature and relative humidity values inside the same group sometimes exceeds the error margins claimed by the datasheets of the sensor. This is possible as these sensors are low-cost and may present in some cases larger errors. However, these values can be corrected and the sensors can be calibrated regularly. On the other hand, the RMSE between groups stays high due to the fact that each group had a different placement and orientation. All these results show that our nodes perform well and confirm again the impact of wind direction and angle of sun exposure on the sensors.

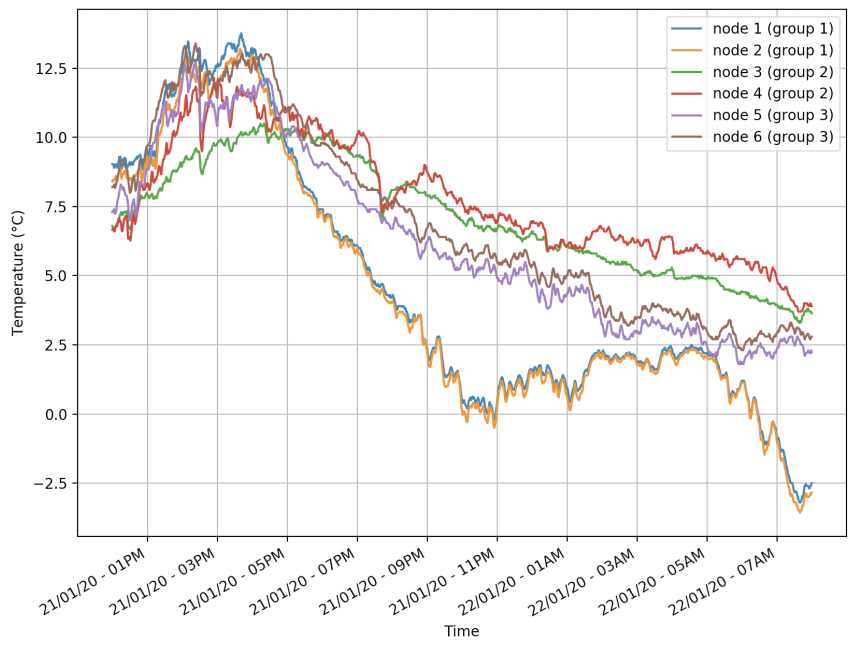

Fig. 10. Temperature measurements from our designed nodes

2) Particulate matter: In the case of particulate matter, Figure 11 presents $\mathrm{PM}_{2.5}$ concentrations from the six nodes. For readability reasons, measurements have been aggregated into $30 \mathrm{~min}$ averages in this plot. However, all calculations were based on 1 min averages. We can observe that PM concentration values from all six nodes present the same trend and a good correlation during the whole period of the test, excepting node 6 which recorded lower concentrations than the others.

Another interesting result presented in Table IIII is that unlike temperature and relative humidity, the RMSE and the coefficient of correlation for $\mathrm{PM}_{2.5}$ and $\mathrm{PM}_{10}$ concentrations have reasonable values regardless of the sun exposure of the nodes or their placement. This indicates that the impact of the sun and wind direction is lower on PM concentrations than temperature and humidity. It has to be noted that the distance separating the sensor nodes was just few meters as they were deployed on the rooftop of the same building. 


\begin{tabular}{|c|c|c|c|c|c|c|c|c|c|}
\hline & \multirow{2}{*}{ Group number } & \multicolumn{2}{|c|}{ Temperature } & \multicolumn{2}{|c|}{ Relative humidity } & \multicolumn{2}{|c|}{ PM 2.5} & \multicolumn{2}{|c|}{ PM 10} \\
\hline & & RMSE & $\begin{array}{l}\text { Pearson's } \\
\text { coefficient }\end{array}$ & RMSE & $\begin{array}{l}\text { Pearson's } \\
\text { coefficient }\end{array}$ & RMSE & $\begin{array}{l}\text { Pearson's } \\
\text { coefficient }\end{array}$ & RMSE & $\begin{array}{l}\text { Pearson's } \\
\text { coefficient }\end{array}$ \\
\hline \multirow{3}{*}{ Intra-group } & 1 & $0.30{ }^{\circ} \mathrm{C}$ & 0.99 & $10.17 \%$ & 0.99 & $1.28 \mu \mathrm{g} / \mathrm{m}^{3}$ & 0.99 & $1.66 \mu \mathrm{g} / \mathrm{m}^{3}$ & 0.99 \\
\hline & 2 & $0.87^{\circ} \mathrm{C}$ & 0.96 & $5.11 \%$ & 0.95 & $4.11 \mu \mathrm{g} / \mathrm{m}^{3}$ & 0.96 & $5.25 \mu \mathrm{g} / \mathrm{m}^{3}$ & 0.96 \\
\hline & 3 & $0.71{ }^{\circ} \mathrm{C}$ & 0.99 & $13.65 \%$ & 0.99 & $4.73 \mu \mathrm{g} / \mathrm{m}^{3}$ & 0.96 & $5.58 \mu \mathrm{g} / \mathrm{m}^{3}$ & 0.96 \\
\hline \multirow{3}{*}{ Inter-group } & $1-2$ & $4.21{ }^{\circ} \mathrm{C}$ & 0.85 & $15.13 \%$ & 0.92 & $2.02 \mu \mathrm{g} / \mathrm{m}^{3}$ & 0.97 & $2.53 \mu \mathrm{g} / \mathrm{m}^{3}$ & 0.97 \\
\hline & $1-3$ & $2.84{ }^{\circ} \mathrm{C}$ & 0.93 & $9.62 \%$ & 0.95 & $3.80 \mu \mathrm{g} / \mathrm{m}^{3}$ & 0.98 & $4.77 \mu \mathrm{g} / \mathrm{m}^{3}$ & 0.98 \\
\hline & $2-3$ & $1.75^{\circ} \mathrm{C}$ & 0.93 & $9.01 \%$ & 0.97 & $3.59 \mu \mathrm{g} / \mathrm{m}^{3}$ & 0.97 & $4.49 \mu \mathrm{g} / \mathrm{m}^{3}$ & 0.97 \\
\hline
\end{tabular}

RMSE AND CORRELATION COEFFICIENT FOR MEASUREMENTS OF TEMPERATURE, RELATIVE HUMIDITY AND PARTICULATE MATTER CONCENTRATIONS

\begin{tabular}{|l|c|c|c|c|c|c|}
\hline Configuration & $\mathrm{NO}_{2}$ & Temperature and RH & PM sensor & Sampling frequency & Transmission frequency & Average energy consumption \\
\hline Config 1 & $\mathrm{X}$ & $\mathrm{X}$ & $\mathrm{X}$ & $20 \mathrm{sec}$ & $1 \mathrm{~min}$ & $231 \mathrm{~mA}$ \\
\hline Config 2 & $\mathrm{X}$ & $\mathrm{X}$ & $\mathrm{X}$ & $1 \mathrm{~min}$ & $3 \mathrm{~min}$ & $224 \mathrm{~mA}$ \\
\hline Config 3 & $\mathrm{X}$ & $\mathrm{X}$ & - & $20 \mathrm{sec}$ & $1 \mathrm{~min}$ & $115 \mathrm{~mA}$ \\
\hline Config 4 & $\mathrm{X}$ & $\mathrm{X}$ & Without fan & $20 \mathrm{sec}$ & $1 \mathrm{~min}$ & $154 \mathrm{~mA}$ \\
\hline
\end{tabular}

COMPARISON OF DIFFERENT OPERATING CONFIGURATIONS

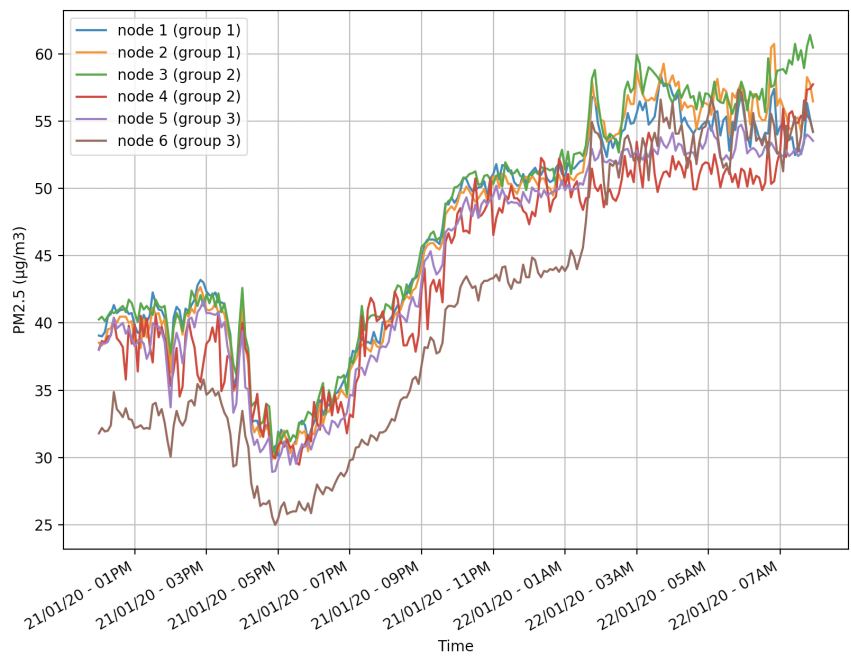

Fig. 11. $\mathrm{PM}_{2.5}$ concentrations measured by our designed nodes

\section{Power consumption}

Energy consumption is of great importance in low-cost WSNs. In fact, Energy requirements differ from one application to another. For our case, we have conducted multiple tests with different configurations to evaluate the power consumption and also to determine which configuration or sensor is power consuming. Results are reported in Table IV As can be expected, the higher the sampling frequency, the higher the energy consumption. The interesting observation is that dividing the sampling frequency by 3 , from one sample every $20 \mathrm{sec}$ (Config 1 ) to one every minute (Config 2), hardly reduces the consumption by $8 \mathrm{~mA}$, around $3.5 \%$. It is worth mentioning that the sensors are not turned off between measuring cycles because the convergence time of some sensors is larger than the chosen sampling period. Indeed the convergence time of the DHT22 is about 2 seconds [40], while the PM sensor needs at least 30 seconds after power-on to start giving reliable results [39]. On the other hand, the required convergence time is more important for the Alphasense $\mathrm{NO}_{2}$ and may reach around ten minutes (see more details in future work section). Another interesting result is the energy consumption of the PM sensor: turning it off (Config 3) reduces the energy consumption by almost half (from $231 \mathrm{~mA}$ to $115 \mathrm{~mA}$ ). This is due to its integrated fan used to aspire the airflow. In the last test (Config 4), the fan of the PM sensor was turned off. The energy consumption droped to $154 \mathrm{~mA}$, meaning that the fan consumes $77 \mathrm{~mA}$ while the electronics of the Grove PM sensor uses $39 \mathrm{~mA}$. Nevertheless, in order to obtain reliable measurement data, it is recommended to use the fan of the sensor.

Based on these tests we choose the first configuration, i.e. maintaining the sampling frequency at 20 seconds and sending data every minute. This will guarantee a good temporal and spatial resolution with an estimated life time of 22 hours using a $5100 \mathrm{mAh}$ battery. We believe that this is a good battery life since our participatory measurement campaigns last about two hours.

\section{FUTURE WORK}

Through this project, we were able to design and develop a general IoT platform, and to test it on air quality monitoring application. This could not have been achieved without addressing multiple challenges related to both IoT and air quality. Nevertheless, multiple challenges still need to be addressed in this field. Going in that direction, we are planning to investigate the following issues.

\section{A. Sensors' convergence time and sensing duty cycling}

WSN are often limited by their resources, especially when it is about energy. To cope with that, we usually adopt some solutions such as the sensing and the radio duty cycling. The sensing duty cycling consists of putting the sensors in sleep mode unless they are performing measurements. Such duty cycle behavior is limited by the convergence time of the sensors embedded in the node. The convergence time is indeed the duration that a sensor needs in order to reach a steady state in stationary conditions and then to output valid readings. Every sensor has its own stabilization time that depends not only on the type of the probe but may also depend on the 
duration of the sleep phase. Based on the datasheets, the convergence time of the DHT22 is about 2 seconds [40], while the PM sensor convergence time is about 30 seconds [39]. We have conducted first experiments to estimate the convergence time of Alphasense NO2 sensor in stationary conditions and we found that this time ranges from around 50 seconds to ten minutes when the previous sleep phase goes from one minute to one hour respectively.

We are planning to conduct extensive experiments to assess the impact of sleeping time on the convergence one for the different used sensors. This would help us to propose adequate sensing duty cycling approaches in order to enhance the node lifetime while keeping valid measured data.

\section{B. Sampling scheduling}

In the case of battery-powered WSNs, generally, the nodes perform measurements at well-defined time intervals in order to extend the battery life. Though, it is always better to have small time intervals especially in air quality monitoring to improve spatio-temporal resolution and data quality. Currently, our nodes perform their measurements every 20 seconds and then send data after each 1 minute. We want to evaluate the loss in terms of mapping quality when considering higher sensing intervals.

Indeed, we have recently reconsidered and compared some regression approaches to assimilation ones while taking into account the intrinsic characteristics of dense deployment of low-cost WSN for air quality monitoring (high density, numerical model errors and sensing errors) [56]. We have proposed a general framework that allows the comparison of different strategies based on numerical simulations and adequate estimation of the simulation error covariances as well as the sensing errors covariances. In addition, we have conducted extensive simulations based on a widely used numerical model and the characterization of the simulation errors.

While using the results of this work [56] and realistic data that we collect in our measurement campaigns, we want to study the impact of the sampling frequency on the overall air quality mapping while using different regression and assimilation approaches. A first simple comparison should be done between 20 -second interval data, 40-second interval data, 60 -second interval data, etc. This should assess the trade-off between sampling frequency and air quality mapping using mobile low-cost sensors.

In this part, we want also to conduct more analysis in order to propose adaptive sampling scheduling in function of the node's trajectory and speed, the sensing quality, etc. this may lead to irregular sampling intervals in order to enhance the node's lifetime while enhancing the overall air quality estimation. We also believe that it would be interesting to see if we can turn off the GPS receiver at some sensing cycles and then try to predict the position in which a measure was taken based on the time of the measure and its value. This would make it possible to extend the battery life of the node even more.

\section{Improve the platform}

Our platform is operational and is currently used in several measurement campaigns in Lyon (around ten campaigns until now). However, we believe that there is still room for improvement. To this aim, we are planning to improve our platform by adding the possibility to remotely manage the nodes. This will give us the ability to change the sampling/transmission parameters of the nodes and to put a certain sensor/receiver in sleep mode. Therefore, the web application will not only be used for data visualization but also for nodes administration. Regarding the node, we could add BLE support, this will give us the ability to use, when possible, the GPS of smartphones instead of the integrated GPS module, which could save us some energy. Another possible improvement is the support of downloading sensor data by just connecting the node to a PC or a smartphone via BLE.

\section{CONCLUSIONS}

Today, climate change is a global challenge and represents a serious concern. As a result, a fine knowledge of air quality and urban heat islands is strongly recommended to implement appropriate policies. To tackle this challenge, low-cost environmental WSNs are increasingly present and integrated in smart cities solutions. They offer new possibilities of monitoring air quality and urban heat islands, reduce the deployment and maintenance cost, and improve the spatiotemporal resolution.

In this work, we present a mobile participatory-based air quality and urban heat islands monitoring platform. It takes advantage of the low-cost and high accessibility of environmental sensors and long range communication technologies to offer high data availability and spatio-temporal resolution. We have designed small, mobile and modular sensor nodes that can be used not only for air quality applications, but in other environmental applications as well. Furthermore, we have designed an intuitive web interface to visualize sensor data, and have made collaboration between our platform and third party services possible. To validate the platform, we have carried out multiple tests involving reliable reference stations and sensors. The results were promising and it was shown that our nodes can be used in the field and thus contribute to the improvement of the knowledge of air quality and urban heat islands.

\section{ACKNOWLEDGMENT}

This work has been supported by the "LABEX IMU" (ANR10-LABX-0088) of Université de Lyon, within the program "Investissements d'Avenir" (ANR-11-IDEX-0007) operated by the French National Research Agency (ANR).

\section{REFERENCES}

[1] World Health Organization, "Burden of disease from the joint effects of household and ambient air pollution for 2016," 2018. [Online]. Available: https://www.who.int/airpollution/data/AP_joint_effect_BoD_ results_May2018.pdf

[2] G. Cannistraro, M. Cannistraro, A. Cannistraro, A. Galvagno, and F. Engineer, "Analysis of air pollution in the urban center of four cities sicilian," Int. J. Heat Technol, vol. 34, pp. S219-S225, 2016. 
[3] R. Basu and J. M. Samet, "Relation between elevated ambient temperature and mortality: a review of the epidemiologic evidence," Epidemiologic reviews, vol. 24, no. 2, pp. 190-202, 2002.

[4] L.-W. Lai and W.-L. Cheng, "Air quality influenced by urban heat island coupled with synoptic weather patterns," Science of the total environment, vol. 407, no. 8, pp. 2724-2733, 2009.

[5] Y.-H. Ryu, J.-J. Baik, and S.-H. Lee, "Effects of anthropogenic heat on ozone air quality in a megacity," Atmospheric environment, vol. 80, pp. 20-30, 2013.

[6] P. Kumar, L. Morawska, C. Martani, G. Biskos, M. Neophytou, S. Di Sabatino, M. Bell, L. Norford, and R. Britter, "The rise of low-cost sensing for managing air pollution in cities," Environment international, vol. 75, pp. 199-205, 2015.

[7] N. Castell, F. R. Dauge, P. Schneider, M. Vogt, U. Lerner, B. Fishbain, D. Broday, and A. Bartonova, "Can commercial low-cost sensor platforms contribute to air quality monitoring and exposure estimates?" Environment international, vol. 99, pp. 293-302, 2017.

[8] M. Gerboles, A. Borowiak, and L. Spinelle, "Measuring air pollution with low-cost sensors," 2017.

[9] R. K. Ganti, F. Ye, and H. Lei, "Mobile crowdsensing: current state and future challenges," IEEE communications Magazine, vol. 49, no. 11, pp. 32-39, 2011.

[10] M. Agarwal and A. Tandon, "Modeling of the urban heat island in the form of mesoscale wind and of its effect on air pollution dispersal," Applied Mathematical Modelling, vol. 34, no. 9, pp. 2520-2530, 2010

[11] S. Ferdoush and X. Li, "Wireless sensor network system design using raspberry pi and arduino for environmental monitoring applications," Procedia Computer Science, vol. 34, pp. 103-110, 2014.

[12] L. Lombardo, S. Corbellini, M. Parvis, A. Elsayed, E. Angelini, and S. Grassini, "Wireless sensor network for distributed environmental monitoring," IEEE Transactions on Instrumentation and Measurement, vol. 67 , no. 5, pp. 1214-1222, 2017.

[13] P. Arroyo, J. L. Herrero, J. I. Suárez, and J. Lozano, "Wireless sensor network combined with cloud computing for air quality monitoring," Sensors, vol. 19, no. 3, p. 691, 2019

[14] N. Castell, M. Kobernus, H.-Y. Liu, P. Schneider, W. Lahoz, A. J. Berre, and J. Noll, "Mobile technologies and services for environmental monitoring: The citi-sense-mob approach," Urban climate, vol. 14, pp. 370-382, 2015

[15] Y. Zhuang, F. Lin, E.-H. Yoo, and W. Xu, "Airsense: A portable contextsensing device for personal air quality monitoring," in Proceedings of the 2015 Workshop on Pervasive Wireless Healthcare. ACM, 2015, pp. $17-22$.

[16] A. Anjomshoaa, F. Duarte, D. Rennings, T. J. Matarazzo, P. deSouza, and C. Ratti, "City scanner: Building and scheduling a mobile sensing platform for smart city services," IEEE Internet of Things Journal, vol. 5, no. 6, pp. 4567-4579, 2018.

[17] A. Boubrima, "Deployment and scheduling of wireless sensor networks for air pollution monitoring," Ph.D. dissertation, University of Lyon, 2019.

[18] D. Hasenfratz, O. Saukh, C. Walser, C. Hueglin, M. Fierz, and L. Thiele, "Pushing the spatio-temporal resolution limit of urban air pollution maps," in 2014 IEEE International Conference on Pervasive Computing and Communications (PerCom). IEEE, 2014, pp. 69-77.

[19] C. A. Pope III, R. T. Burnett, M. J. Thun, E. E. Calle, D. Krewski, K. Ito, and G. D. Thurston, "Lung cancer, cardiopulmonary mortality, and long-term exposure to fine particulate air pollution," Jama, vol. 287, no. 9, pp. 1132-1141, 2002.

[20] G. Bowatte, C. Lodge, A. J. Lowe, B. Erbas, J. Perret, M. J. Abramson, M. Matheson, and S. C. Dharmage, "The influence of childhood trafficrelated air pollution exposure on asthma, allergy and sensitization: a systematic review and a meta-analysis of birth cohort studies," Allergy, vol. 70, no. 3, pp. 245-256, 2015.

[21] E. P. Agency, "Air pollution: Current and future challenges," 2018. [Online]. Available: https://www.epa.gov/clean-air-act-overview/ air-pollution-current-and-future-challenges

[22] B. Xu and B. Lin, "How industrialization and urbanization process impacts on co2 emissions in china: Evidence from nonparametric additive regression models," Energy Economics, vol. 48, pp. 188 - 202, 2015. [Online]. Available: http://www.sciencedirect.com/science/article/ pii/S0140988315000195

[23] R. Paranunzio, S. Ceola, F. Laio, and A. Montanari, "Evaluating the effects of urbanization evolution on air temperature trends using nightlight satellite data," Atmosphere, vol. 10, no. 3, 2019. [Online]. Available: https://www.mdpi.com/2073-4433/10/3/117

[24] "Changement climatique en rhône-alpes. températures moyennes annuelles et saisonnières," 2016. [Online].
Available: https://www.orcae-auvergne-rhone-alpes.fr/fileadmin/ user_upload/mediatheque/ORCAE/Documents/Publications/ORECC_ FicheIndicateur2017_Temperaturesmoyennes_V20170929.pdf

[25] F. Renard, L. Alonso, Y. Fitts, A. Hadjiosif, and J. Comby, "Evaluation of the effect of urban redevelopment on surface urban heat islands," Remote Sensing, vol. 11, no. 3, p. 299, 2019.

[26] M. Al-Azzawi and R. Raeside, "Modeling pedestrian walking speeds on sidewalks," Journal of Urban Planning and Development, vol. 133, no. 3, pp. 211-219, 2007

[27] R. Rastogi, S. Chandra et al., "Pedestrian flow characteristics for different pedestrian facilities and situations," 2013.

[28] K. Kotkar, R. Rastogi, and S. Chandra, "Pedestrian flow characteristics in mixed flow conditions," Journal of Urban Planning and Development, ASCE, vol. 136, no. 3, pp. 23-33, 2010.

[29] "The things network." [Online]. Available: https://www. thethingsnetwork.org/

[30] LoRaWAN TM 1.0.3 Specification, LoRa Alliance, Inc, 72018.

[31] M. C. Bor, J. Vidler, and U. Roedig, "Lora for the internet of things." in $E W S N$, vol. 16, 2016, pp. 361-366.

[32] LoRaWAN® Regional parameters, LoRa Alliance, Inc, 22020.

[33] N. Blenn and F. Kuipers, "Lorawan in the wild: Measurements from the things network," arXiv preprint arXiv:1706.03086, 2017.

[34] N. Zinas, S. Kontogiannis, G. Kokkonis, S. Valsamidis, and I. Kazanidis, "Proposed open source architecture for long range monitoring. the case study of cattle tracking at pogoniani," in Proceedings of the 21st PanHellenic Conference on Informatics. ACM, 2017, p. 57.

[35] "Placepod." [Online]. Available: https://www.pnicorp.com/placepod/

[36] "Vinduino r3 sensor station." [Online]. Available: https://www. thethingsnetwork.org/marketplace/product/vinduino-r3-sensor-station

[37] Alphasense Ltd, "No2-b43f nitrogen dioxide sensor." [Online]. Available: http://www.alphasense.com/WEB1213/wp-content/uploads/ 2019/09/NO2-B43F.pdf

[38] H. Price, "Air analysis I field portable instruments for the measurement of airborne hazards," in Encyclopedia of Analytical Science (Third Edition), P. Worsfold, C. Poole, A. Townshend, and M. Miró, Eds. Oxford: Academic Press, 2019, pp. 40 - 43. [Online]. Available: http: //www.sciencedirect.com/science/article/pii/B9780124095472126800

[39] Shenzhen Co., Ltd, "Hm-3300/3600," $2018 . \quad$ [Online]. Available: https://github.com/SeeedDocument/Grove-Laser_PM2.5_ Sensor-HM3301/raw/master/res/HM-3300\%263600_V2.1.pdf

[40] Aosong Electronics O., Ltd, "Digital-output relative humidity \& temperature sensor/module dht22," 2019. [Online]. Available: https: //www.sparkfun.com/datasheets/Sensors/Temperature/DHT22.pdf

[41] Microchip Technology Inc, "Sam d21 family," 2018. [Online]. Available: https://www.mouser.fr/pdfdocs/ SAM-D21-Family-Datasheet-DS40001882C.pdf

[42] Murata Investment Co., Ltd, "Sub-g module data sheet," 2018. [Online]. Available: https://wireless.murata.com/datasheet?/RFM/data/ type_abz.pdf

[43] Adafruit learning system, "Adafruit 4-channel adc breakouts," 2019. [Online]. Available: https://cdn-learn.adafruit.com/downloads/ pdf/adafruit-4-channel-adc-breakouts.pdf?timestamp=1575440557

[44] GlobalTop Technology Inc., "Fgpmmopa6h," 2011. [Online]. Available: https://cdn-shop.adafruit.com/datasheets/ GlobalTop-FGPMMOPA6H-Datasheet-V0A.pdf

[45] Adafruit learning system, "Adafruit powerboost 1000c," 2019. [Online]. Available: https://cdn-learn.adafruit.com/downloads/ pdf/adafruit-powerboost-1000c-load-share-usb-charge-boost.pdf? timestamp $=1575440467$

[46] A. Augustin, J. Yi, T. Clausen, and W. M. Townsley, "A study of lora: Long range \& low power networks for the internet of things," Sensors, vol. 16, no. 9, p. 1466,2016

[47] E. L. Lehmann and H. J. D'Abrera, Nonparametrics: statistical methods based on ranks. Holden-Day, 1975.

[48] Y. K. Cheung and J. H. Klotz, "The mann whitney wilcoxon distribution using linked lists," Statistica Sinica, pp. 805-813, 1997.

[49] Dostmann electronic $\mathrm{GmbH}$, "Log 32 th pdf- data logger for temperature and humidity." [Online]. Available: https://dostmann-electronic.de/ product/log-32-th-pdf-data-logger-for-temperature-and-humidity.html

[50] TFA Dostmann, "Protective cover for outdoor transmitter." [Online]. Available: https://www.tfa-dostmann.de/en/produkt/ protective-cover-for-outdoor-transmitter/

[51] Davis Instruments, "Radiation shield(7714) specification sheets." [Online]. Available: https://www.davisinstruments.com/product_documents/ weather/spec_sheets/DS7714_6838_Rad\%20Shields_Spec_Sheet.pdf 
[52] M. Schumacher, "Two-sample tests of cramér-von mises-and kolmogorov-smirnov-type for randomly censored data," International Statistical Review/Revue Internationale de Statistique, pp. 263-281, 1984.

[53] W. H. Kruskal and W. A. Wallis, "Use of ranks in one-criterion variance analysis," Journal of the American statistical Association, vol. 47, no. 260, pp. 583-621, 1952.

[54] F. Delaine, B. Lebental, and H. Rivano, "In situ calibration algorithms for environmental sensor networks: A review," IEEE Sensors Journal, vol. 19, no. 15, pp. 5968-5978, 2019.

[55] B. Maag, Z. Zhou, and L. Thiele, "A survey on sensor calibration in air pollution monitoring deployments," IEEE Internet of Things Journal, vol. 5, no. 6, pp. 4857-4870, 2018.

[56] M. A. Fekih, I. Mokhtari, W. Bechkit, Y. Belbaki, and H. Rivano, "On the regression and assimilation for air quality mapping using dense low-cost wsn," in International Conference on Advanced Information Networking and Applications. Springer, 2020, pp. 566-578.

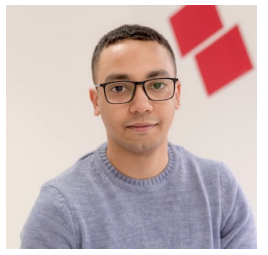

Mohamed Anis Fekih is a PhD student at INRIA, France and a member of the Agora team within the CITI Lab of INSA-Lyon. He received his master and engineering degrees in Computer Science with highest distinction from the "Ecole nationale Supérieure d'Informatique", Algiers in 2018. His $\mathrm{PhD}$ thesis is part of the French interdisciplinary project « 3M'Air », which focuses on the use of mobile participatory monitoring of air pollution and urban heat islands. His research interests include IoT platforms, spatio-temporal air quality mapping, and path planning applied to mobile crowdsensing.

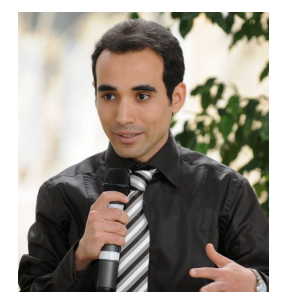

Walid Bechkit is an associate professor within the Telecommunications department of INSA-Lyon, France. He is also a member of the INRIA Agora team of the CITI laboratory. He obtained his $\mathrm{Ph} . \mathrm{D}$. in System and Information Technology from the Compiegne University of Technology (U.T.C.), France in 2012 and his engineering degree in Computer Science from the "Ecole nationale Supérieure d'Informatique", Algiers in 2009. His main research interests include deployment, reliability, security and energy saving issues in wireless sensor networks.

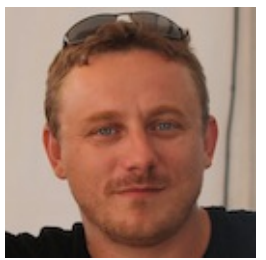

Hervé Rivano is a full professor at INSA-Lyon, France and the head of the INRIA Agora team, which focuses on the use of wireless networks in smart cities. Prior to that, he had been an INRIA and CNRS researcher from October 2004 to August 2017. He obtained his PhD in November 2003 from the University of Nice-Sophia Antipolis after graduated from the "Ecole Normale Supérieure de Lyon", France. His research interests include combinatorial optimization and approximation algorithms applied to network design and provisioning.

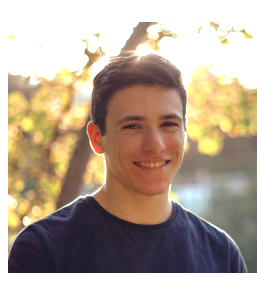

Manoël Dahan is a Telecommunications Engineer trained at INSA Lyon. He joined the AGORA team of the CITI laboratory following his graduation. Missioned in support of the UrPolSens project, he developed a fleet of battery-powered nodes measuring NO2. Following a deployment in the city of Lyon, he is recruited in the same team for a European project of smart lighting (Interreg eSmartCity) for the design of nodes allowing the detection of activity in the streets. This system provides a database leading to the identification of the benefit of an smart lighting system deployment. As part of the 3M'air project, it also participates in the development of sensors measuring pollution for use by pedestrians.

PLACE

PHOTO

HERE

Florent Renard is an associate professor in Geography and Spatial Planning at the University of Lyon. $\mathrm{He}$ is a researcher at the UMR CNRS Environment City Society, a member of the Labex Intelligence des Mondes Urbains (IMU) and of the Field Observatory for Water Management (OTHU). His main research activities focus on the problems of heat islands, microclimates and thermal comfort in urban areas, the local impacts of climate change, particularly in terms of temperature and rainfall, territorial vulnerabilities and natural risk management. In a complementary way, he is also involved in some aspects of the geography of health, such as the emergency management of stroke and the subsequent territorial organization or the spatial socio-economic determinants of this pathology. The research work carried out combines mixed techniques, both quantitative and qualitative, with the use of spatial analysis and modelling by GIS, satellite imagery or weather radar by remote sensing, semi-directed interviews and participatory science. He is also in charge of the Environmental Management mention and the Master's degree in Environmental Geosystems at the University of Lyon (France).

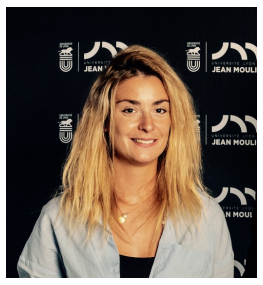

Lucille Alonso is a $\mathrm{PhD}$ student at University of Lyon and a member of the UMR 5600 CNRS "Environment City and Society" research unnit. She works mainly on urban climate monitoring and modelling using satellite data in France, on the Lyon metropolitan area, and the assessing of socio-economic and physiological vulnerabilities of populations to heatwaves. Her research interests include remote sensing, spatial analysis for land use/land cover analysis and modelling, urban environment monitoring and planning, and vulnerabilities to heatwaves.

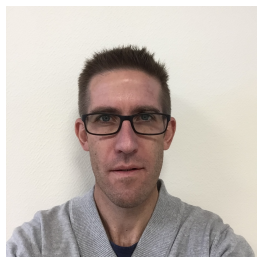

Florent Pineau is a Research Engineer in the Air Quality Laboratory at the Oil \& Gas Company TOTAL. After obtaining Diplomas in Biochemistry and Biology analysis at the University of Angers and in Health and Environmental Science at the University of Lille, Florent Pineau obtained a Master in Occupational and Environmental hygiene at the CNAM (Conservatoire National des Arts et Métiers) in 2005. His current research focuses on the quantification of pollutants by combining measurement from various technologies (optical; punctual, monitoring network) to reverse modelling approach. Florent participates to the elaboration of microsensors networks coupled with weather station to detect fugitive / diffuse emission by remote sensing technologies in order to quantify emission using reverse modelling approach. 